\title{
A Software Based DVB-T2 Time Frequency Slicing Evaluation Framework
}

\author{
Morgade J., Angueira P., Arenas J. A., Basterra A. \\ Dpt. Of Communications Engineering (KIS) \\ University of Basque Country (UPV/EHU) \\ Bilbao, Spain \\ Alda. Urquijo,s/n, 48013 \\ Email: javier.morgade@ehu.es
}

\author{
Plets D., Joseph W. \\ Wireless and Cable-IBBT, Dept. of Information Technology \\ Ghent University Technology \\ Ghent, Belgium \\ Email: wout.joseph@intec.Ugent.be
}

\begin{abstract}
DVB-T2 is the second generation terrestrial broadcast system developed within the DVB consortium. Based on multi-carrier transmission OFDM, DVB-T2 introduces many innovative tools offering high flexibility to broadcasters. Among many other new techniques DVB-T2 defines an optional TimeFrequency-Slicing (TFS) profile. In TFS slices of a PLP are sent over multiple and non necessarily adjacent RF frequency channels. This technique provides a gain associated to propagation diversity, as well as an intrinsic multiplexation gain. Following a software defined radio approach this paper presents a flexible DVB-T2 Time Frequency Slicing evaluation framework built to study the practical performance of DVB-T2 Time Frequency Slicing configurations under realistic channel scenarios. The paper focuses on the diversity gain associated to propagation diversity.
\end{abstract}

Keywords—dvb-t2; tfs; plps, sdr-radio;

\section{INTRODUCTION}

The DVB-T2 standard is the second generation standard for terrestrial delivery produced by the DVB (Digital Video Broadcasting) consortium [1] . The standard was originated by the demands to increase the spectral efficiency of digital terrestrial broadcast systems in the VHF/UHF bands. The standard provides a remarkable flexibility in multiplex allocation, coding, modulation and RF parameters. This flexibility allows bitrates that range from a few Mbps to $50 \mathrm{Mbps}$ depending on the system robustness and associated throughput tradeoff. DVB-T2 is based on Orthogonal frequency division multiplexing (OFDM), however, in addition to the original DVB-T $2 \mathrm{~K}$ and $8 \mathrm{~K}$ modes, new FFT sizes of $1 \mathrm{~K}, 4 \mathrm{~K}, 16 \mathrm{~K}$ and $32 \mathrm{~K}$ subcarriers have been defined. Moreover, higher data rates are possible with 256QAM constellations. Regarding channel coding, DVB-T2 inherits the FEC coding from DVB-S2 based on a concatenation of LDPC and $\mathrm{BCH}$ codes [2]

In DVB-T2, OFDM symbols are arranged on a sequence of T2 frames where future extensions frames (FEFs) have been also included in the standard in order to allow the introduction of future services like T2-Lite, DVB-NGH or future options that might include Multiple-Input-Multiple-Ouput (MIMO) antenna systems or Time Frequency Slicing profiles. Another remarkable improvement of DVB-T2 comes from the ability to define service specific robust levels. With the Physical Layer Pipes (PLPs) different protection levels can be configured in DVB-T2, including the channel coding parameters, constellation orders and interleaving depths.

Time Frequency Slicing (TFS) is one of the novel tools available as an option in the DVB-T2 standard [1]. In TFS multiple RF channels can be combined into a wider virtual channel where in addition to the augmented overall bit rate, an improvement of the system frequency diversity can be achieved [1]. These advantages are achieved at the expense of increasing receiver implementation complexity. TFS would require at least two tuners or at least a frequency hopping receiving scheme. Additional restrictions regarding the minimum hopping time between frequency channels is also a challenge to be addressed. Despite of all these restrictions, TFS would increase significantly the overall DVB-T2 system performance, providing a valuable statistical multiplexing gain that would add to the achievable network gain associated to the improved robustness against time-varying channels and interference [3][4].

There have been few previous publications in this area. In [5] the flexibility of time interleaving mechanisms for DVB-T2 is analyzed in deep. Tradeoffs in terms of diversity, latency and power saving are studied but results for TFS are not provided. In [6] the same authors proposed empirical TFS gain figures. However, these results are based on field strength measurements and do not consider the real DVB-T2 system performance. The same approach is employed in [7]. Finally, Makni et.al. [8], present TFS performance simulations under Rayleigh channel conditions and for emulated SNR imbalance values between RF channels. In summary, the results available results up to date do not consider the structure of a real T2 receiver.

In this work, a flexible high performance DVB-T2 Time Frequency Slicing Evaluation Framework is presented. The paper is organized as follows: first sections provide a description of the main characteristics of the T2-TFS profile. The second and third sections contain the system level architecture of the proposed TFS evaluation framework. In the fourth section provides representative simulation results. 


\section{The DvB-T2 Time Frequency Slicing Profile}

Time-Frequency-Slicing (TFS) is a DVB-T2 profile where the sub-slices of a PLP are sent over multiple RF frequencies during the T2-frame. In addition of the common interleaving process applied in single profile with TFS interleaving might be also applied over multiple RF channels.

DVB-T2 has two different types of PLPs depending on the number of time slices per frame configured in the given T2 system. PLPs type 1 are always carried using a single time slice per frame, on the other hand PLPs type 2 can be sent over multiple sub-slices. DVB-T2 also defines common PLPs, containing information associated to other PLPs that share the same group Id [1].

In TFS the T2-frame has a similar structure as the traditional single RF channel signal. However, in TFS there will be some restrictions in the frame length to enable enough time for switching between the different RF channels that convey the single TFS multiplex. Regarding the transmitted information, P1 symbols, main L1 signaling cells and common PLPs are repeated over all RF channels forming the TFS structure. On the contrary, data PLPs are distributed over all RF channels. Moreover, Type 1 and Type 2 PLPs have different treatment. Type 2 PLPs can be carried out over all the sub-slices defined per T2 frame, and therefore, they can jump between RF channels during the $\mathrm{T} 2$ frame. On the contrary, Type 1 PLPs are required to travel on the same RF channel during $\mathrm{T} 2$ frame and the jumping can happen only between $\mathrm{T} 2$ frames. As a consequence, the addressing of PLPs data cells is not straightforward, if compared to the single RF channel DVB-T2 profile. Figure 1 represents the overall T2-frame structure and type PLPs distribution when using 6 RF channels.

The indexing of the RF channels is signaled in the CURRENT RF IDX and in the RF IDX parameter in the configurable part of the L1-post signaling and therefore the signaling part is slightly different in all the TFS branches. Details for a TFS receiver implementation can be found in [1].

\section{SOFTWARE BASED DVB-T2-TIME FREQUENCY SLICING RECEIVER}

In general, when the technology behind a digital broadcasting standard is mature, a number of professional and/or commercial receivers are available from different manufactures. The situation is different when testing emerging technologies like TFS where reference receiver implementations are not available in the market. Following a "software defined radio" approach, a high performance DVBT2 TFS receiver has been developed in order to evaluate properly TFS in DVB-T2.

The receiver is fully compatible with the DVB-T2 standard [1]. It takes as input DVB-T2 IQ (In-phase and Quadrature) baseband samples and performs all the signal resampling, synchronization, channel estimation and equalization processes. A complete BICM decoder per PLP is included where all FEC and interleaving stages are threaded. In addition to the process of decoding of the source stream, the receiver is

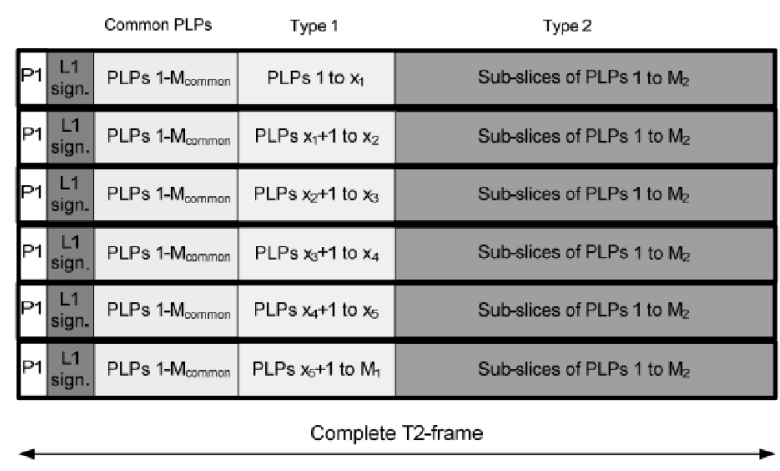

Fig. 1. T2-frame Structure in a TFS System with 6 RF channels [1]

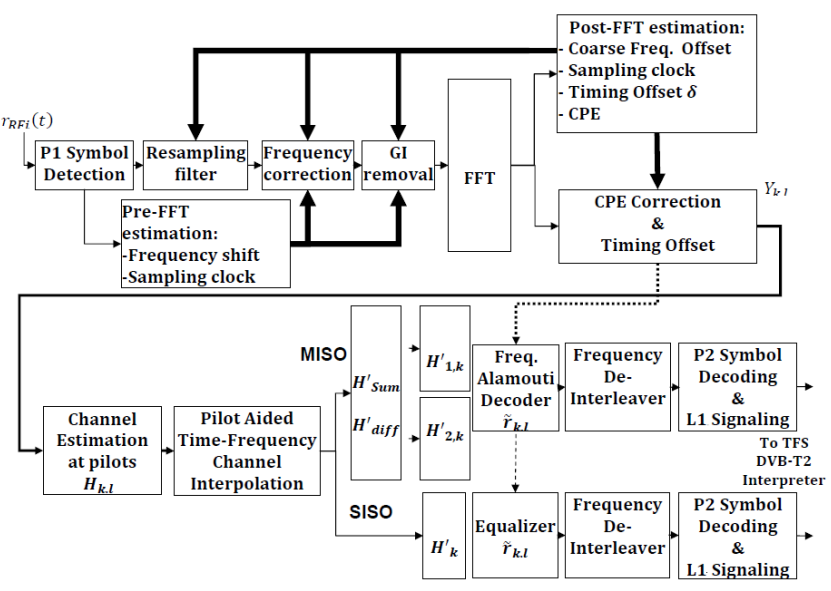

Fig. 2. Per-RF branch receiver model

also able to provide detailed measurements. If compared to the single RF channel DVB-T2 profile, the receiver incorporates a TFS interpreter including the corresponding additional RF branches dealing with required RF channel specific operations. In following sub-sections the high level characteristics of our TFS DVB-T2 receiver will be introduced.

In Fig 2 a detailed architecture of the software backend is depicted. The block diagram summarizes al the signal acquisition, signal synchronization, demodulation and equalization steps. In a similar way to other OFDM receivers, a complete synchronization both in time and frequency is required in DVB-T2. In this sense, in DVB-T2, different signals are particularly designed for the synchronization stages. These are mainly: the P1 symbol, the P2 symbol and scattered and continual pilots. The P1 symbol is repeated at the beginning of every frame. After decoding, this symbol has some signaling information that is used to enable the receiver to indentify the FFT mode, it also signals if the transmitted signal is working in the MISO (Multiple-input-single-output) diversity profile or in the common SISO operation. After a successful decoding of the P1 symbol and based on a guard interval correlation method, the receiver detects the used guard interval. 
$\operatorname{mm} 13-59$

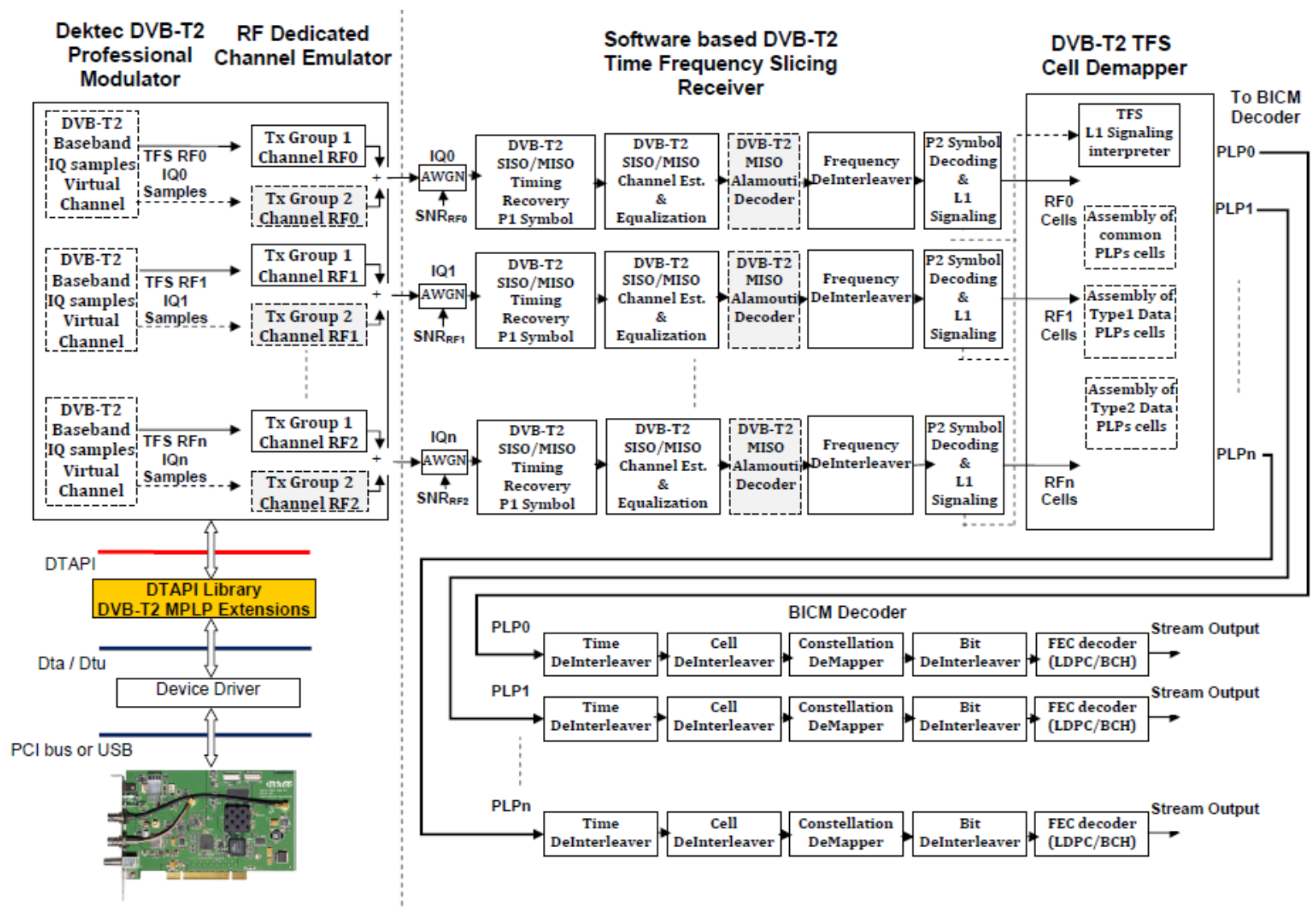

Fig. 3. Software based DVB-T2 Time Frequency Slicing (TFS) Receiver Model

Once the initial synchronization is performed, the received signal has to be continuously tracked in both time and frequency. The fine frequency shift estimation is performed using the guard interval correlation [9]. After the FFT demodulation and guard interval removal, the continual pilots are used for frequency synchronization tasks, being the coarse frequency synchronization, the CPE estimation and clock recovery carried correlating the reference continual pilots against the acquired input signal in the frequency domain.

Even if the input signal is synchronized correctly the output signal after the FFT demodulation might differ with respect to the original transmitted signal. The receiver carrier amplitudes at the output of the FFT demodulator might be affected by the channel through which the signal has been transmitted. In this sense, the receiver includes representative channel estimation and equalization stages for both MISO and SISO profiles

At this point it has to be noted that the presented receiving software backend architecture in figure 2, represents all the usual operations carried out to synchronize and equalize one DVB-T2 signal. For the TFS profile all the signal acquisition, adaptation and equalization stages have been replicated per RF channel. Where a TFS interpreter has been implemented to correctly synchronize the corresponding "virtual RF channels" and therefore be able to perform the corresponding frequency hopping.

\section{MiXed Hw \& SOFTwARE EVALUATION SYSTEM FRAMEWORK}

Evaluating the practical performance of digital broadcast system requires different modules within the system chain. These elements are a transmission system, a channel modeling module and the availability of professional or commercial receivers. However, when testing emerging technologies like TSF limited resources are available on the market. At the time of writing this paper, there are not professional TFS receivers available the market; however there are already available professional solutions to generate real DVB-T2 TFS signals. The framework presented in this paper includes a built-in DVB-T2 TFS receiver implementation as well as a channel emulation module and a mixed hardware/software tool that enables generating TFS signals using equipment from the Dektec manufacturer [10]. The system is shown in Fig. 3.

\section{A. TFS source signals}

DVB-T2-TFS signals are generated with Dektec USB/PCIDVB-T2 professional modulators [10]. Among other features the mentioned modulators support the DVB-T2 Time Frequency Slicing profile, where the corresponding baseband IQ samples per RF channel can be directly written to a file. IQ sample files that can be further feed to the TFS software receiver. It has to be noted at this point that the modulator includes also the ability to simulated fading channels. 


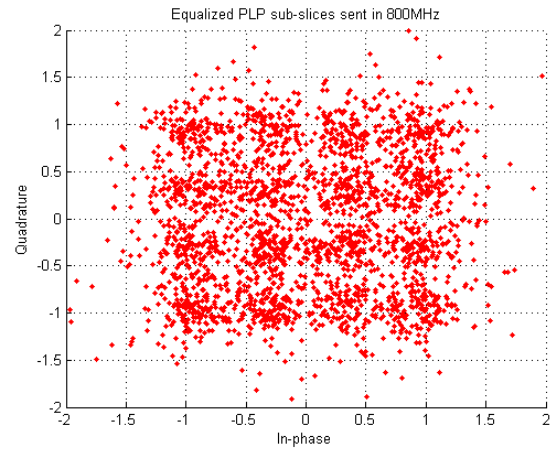

(a)

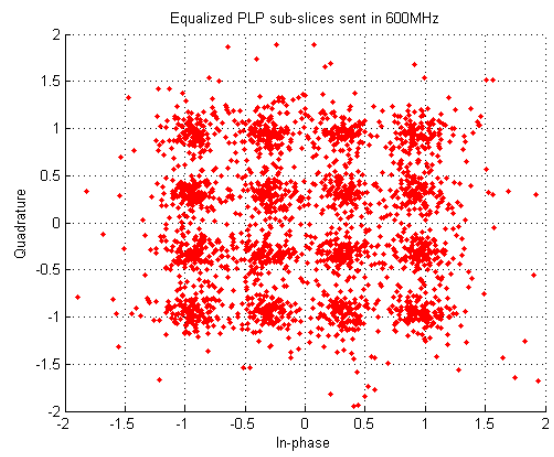

(b)

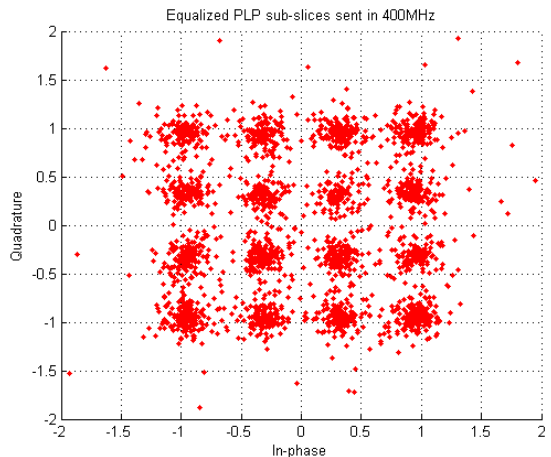

(c)

Fig. 4. 16QAM-2/3 Type2 PLP sent in a T2 TFS Configuration with 3 RF channels $(800,600,400)$ MHz: (a) Equalized sub-slice cells from RF branch1 under TU6 channel@50kmh and $\Delta S N R_{f_{R F i}}=0 \mathrm{~dB}$; (b) Equalized sub-slice cells from RF branch2 under TU6 channel@50kmh and $\Delta S N R_{f_{R F i}}=2.49 \mathrm{~dB} ; ;$ (c) Equalized cells in RF branch3 TU6 channel@50kmh and $\Delta S N R_{f_{R F i}}=6.02 \mathrm{~dB}$

\section{B. TFS channel modelling}

If compared to the single RF channel DVB-T2, the evaluation of the practical performance of Time Frequency Slicing requires taking into consideration the particular characteristics of TFS signals at the receiver input. In practice, TFS might be configured with a number of RF channels that range from 2 channels to 7 . Moreover, the used radio frequency resources might be spread over all the available spectrum range (a significant part of the UHF band). A priori, the main factors affecting the TFS performance are the doppler effect, in both mobile and portable target reception and the power imbalance between RF channels. The simplest approach to characterize the associated propagation conditions could be accomplished tuning the reference channel models available in the literature to characterize the single DVB-T2 profile and adapting its statistical behavior to each RF channel characteristics [4]. For instance the linear dependency between the Doppler frequency shift and the frequency channel for a given speed can be modeled using the well known equation

$$
v(m / s)=f_{\text {doppler }} \cdot c / f_{R F}
$$

where $v$ represent the speed of the target receiver, $f_{\text {doppler }}$ is the experience Doppler shift in $\mathrm{Hz}$ at the given working radio frequency resource $\left(f_{R F}\right)$ and $c$ represents the speed of light in vacuum.

Regarding power imbalance, the approach is based on simulating equivalent noise reception conditions at the input of each "virtual RF channel". A simple way might be using the free-space path loss formula to balance the equivalent noise perception at each "tuner" as follows:

$$
\begin{aligned}
& S N R_{f_{R F i}}(d B)=S N R(d B)+\Delta S N R_{f_{R F i}}(d B) \\
& \Delta S N R_{f_{R F i}}(d B)=20 \cdot \log \left(f_{R F i} / f_{R F m i n}\right)
\end{aligned}
$$

where $\Delta S N R_{f_{R F i}}$ represents the corresponding balance to be applied in decibels over the working RF channel $\left(f_{R F i}\right)$ using the lower frequency resource available for the TFS system under study as reference. Equation (3) can be easily derived from the free-space path loss formula.

\section{SiMULATION RESUlTS}

In order to validate the proposed Time Frequency Slicing evaluation framework, system level simulations have been carried out, where a DVB-T2-LITE, FFT4K, 1/8GI, PP2 system was simulated in both configurations "single profile" and "TFS profile". The single profile was evaluated at $800 \mathrm{MHz}$ where as the TFS profile consisted of a combination of three RF channels. The TFS channels were generated at $800 \mathrm{MHz}$, $600 \mathrm{MHz}$ and $400 \mathrm{MHz}$ respectively. A QAM16-2/3 Type 2 PLP with 6 sub-slices was specifically evaluated to compare the system performance in both single RF and TFS configurations.

Regarding the channel modeling, a reference TU6 channel [4] has been tuned according equation (1) for every RF channel and for the following target receiver speeds $(120,90,50,40$, 20) kmh. Finally, the equivalent $S N R_{f_{R F i}}$ for every RF branch was balanced using the free-space path loss criteria. Finally, the receiver Quasi-error-free condition was measured at the output of the $\mathrm{BCH}$ decoder as the $\%$ of correctly received FEC blocks.

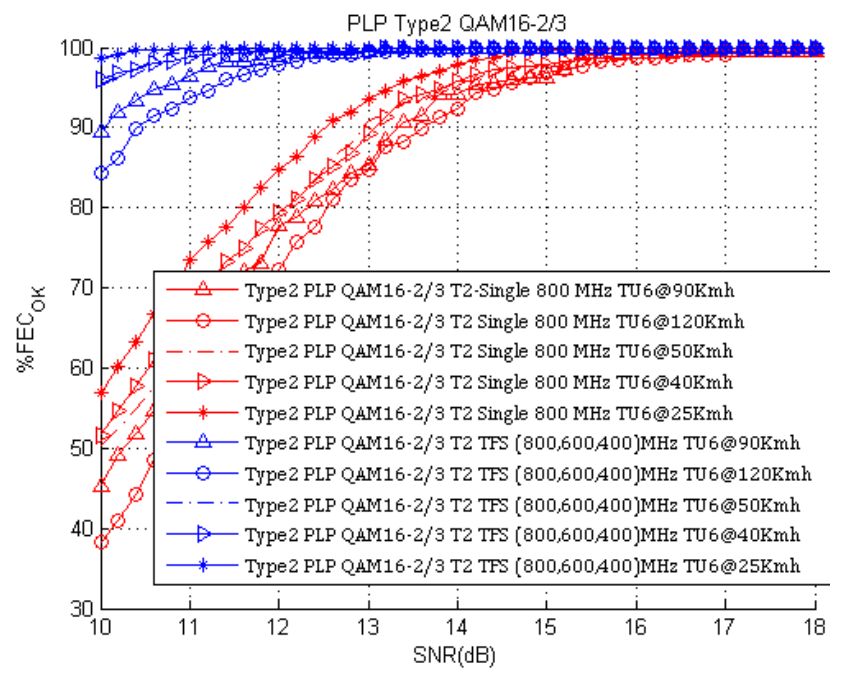

Fig. 5. Simulated TFS gain 
Figure 5 presents the results for both configurations and specific channel conditions. Results show the benefits of TFS and how this profile can improve the overall system performance. Moreover, Figure 4 shows the different degradation levels experienced by PLP when travelling at each radio frequency channels due to the emulated channel conditions.

\section{CONCLUSIONS AND FUTURE WORK}

In this paper a novel Time Frequency slicing evaluation framework has been presented. The framework is composed of different hardware and software subsystems. The transmitting hardware is based on the existing commercial DVB-T2 generation equipment (DTU-215 Gold cards) whereas the receiver is a DVB-T2 custom receiver implementation..

The paper presents also some preliminary results using a TFS channel combination composed by three RF channels evenly distributed over the UHF band. The first simulations show that an average gain of $4 \mathrm{~dB}$ can be achieved for a DVBT2-LITE configuration of FFT4K, 1/8GI, PP2. Provided results are applicable for QAM16-2/3 modulated PLP Type 2 with 6 sub-slices under propagation conditions equivalent to a TU6 channel and with and emulated SNR imbalance between RF channels based on the free-space path loss criteria.

The simulation work continues at the moment of writing this paper, in order to provide TFS gain values for a wider set of DVB-T2 modes as well as extending the propagation conditions using additional channel models, with particular interest on SFN channel models.

\section{REFERENCES}

[1] Digital Video Broadcasting(DVB): Framing Structure, Channel coding and Modulation for digital terrestrial television broadcasting system(DVB-T2), ETSI EN 302755 v1.1.1, September 2009.

[2] Second generation framing structure, channel coding and modulation systems for Broadcasting, Interactive Services, News Gathering and other broadband satellite applications (DVB-S2),ETSI EN 302307 v1.2.1, September 2009.

[3] "Frequency and Network Planning Aspects of DVB-T2", EBUTechnical Report 3348. Geneva, May 2012.

[4] Implementation guidelines for a second generation digital terrestrial television broadcasting system (DVB-T2), TS 102831 V1.2.1 (08/12)

[5] Gozalvez, D.; Gomez-Barquero, D.; Vargas, D.; Cardona, N.; , "Time Diversity in Mobile DVB-T2 Systems," Broadcasting, IEEE Transactions on, vol.57, no.3, pp.617-628, Sept. 2011 doi: 10.1109/TBC.2011.2161189

[6] Gozalvez, D.; Lopez-Sanchez, J.; Gomez-Barquero, D.; Gimenez, J.J.; Cardona, N.; , "Combined Time and Space Diversity: Mobile Reception in DVB-T and DVB-T2 Systems," Vehicular Technology Magazine, IEEE, vol.7, no.4, pp.114-121, Dec. 2012 doi: 0.1109/MVT.2012.2218143

[7] Huuhka, E., et.al.: WP3 - Network \& Channels -B21C Public Deliverable D1 Part 3 - DVBT2 Network Planning, URL: http://projects.celic-initiative/b21c/

[8] Makni, M; Robert, J.; Stare, E.,"Performance Analysis of Time Frequency Slicing", Electronic Media Technology (CEMT), $201114^{\text {th }}$ ITG Conference on. 2011.

[9] Jan-Jaap van de Beek, , Magnus Sandell, and Per Ola B"orjesson, "ML Estimation of Time and Frequency Offset in OFDM Systems

[10] http://www.dektec.com/Products/T2Toolset.asp 